\title{
The Bases of Effective Field Theories
}

\author{
Martin B Einhorn ${ }^{\mathrm{a}, \mathrm{b}}$, José Wudkac \\ ${ }^{a}$ Kavli Institute for Theoretical Physics ${ }^{1}$, University of California, Santa Barbara, CA 93106-4030 \\ ${ }^{b}$ Michigan Center for Theoretical Physics, University of Michigan, Ann Arbor, MI 48109 \\ ${ }^{c}$ Department of Physics and Astronomy, University of California, Riverside, CA 92521-0413
}

\begin{abstract}
With reference to the equivalence theorem, we discuss the selection of basis operators for effective field theories in general. The equivalence relation can be used to partition operators into equivalence classes, from which inequivalent basis operators are selected. These classes can also be identified as containing Potential-Tree-Generated (PTG) operators, Loop-Generated (LG) operators, or both, independently of the specific dynamics of the underlying extended models, so long as it is perturbatively decoupling. For an equivalence class containing both, we argue that the basis operator should be chosen from among the PTG operators, because they may have the largest coefficients. We apply this classification scheme to dimension-six operators in an illustrative Yukawa model as well in the Standard Model (SM). We show that the basis chosen by Grzadkowski et. al. [5] for the SM satisfies this criterion. In this light, we also revisit and verify our earlier result [6] that the dimension-six corrections to the triple-gauge-boson couplings only arise from LG operators, so the magnitude of the coefficients should only be a few parts per thousand of the SM gauge coupling if BSM dynamics respects decoupling. The same is true of the quartic-gauge-boson couplings.
\end{abstract}

Keywords: Effective Lagrangians, Beyond Standard Model, Effective field theory

\footnotetext{
${ }^{1}$ Current address.
} 


\section{Introduction}

Effective quantum field theories have a wide variety of applications in condensed matter [1] and elementary particle physics [2, 3], both as methods for facilitating calculations and as ways of exploring or constraining new physics. In this last application, there has been a revival of interest among high energy physicists since the discovery of a Higgs boson at the CERN LHC, apparently resolving the long-standing uncertainty about the theory of elementary particles known as the Standard Model (SM).

Without knowing the precise form of new degrees of freedom or new particles, there are inherent ambiguities in the form of additional operators to be added to a theory because of the equivalence theorem. As reviewed in more detail below, this states that observable transition amplitudes (S-matrix elements) are unchanged by replacing some operators with others if their difference vanishes "on-shell," i.e., if the difference vanishes when the classical equations of motion (EoM) are satisfied. Although this allows one to reduce the number of new operators and coupling constants that must be introduced [4, 5], in the face of ignorance of the underlying dynamics, it seems the choices are both arbitrary and irrelevant.

In any given model extending the SM to higher energy scales, some operators $Q_{i}$ may arise from tree diagrams in an underlying theory, while others may only emerge from loop corrections. Generally, the coefficients of loop diagrams, as quantum corrections to the classical theory, are perturbatively smaller than those associated with tree diagrams, being associated with higher powers of dimensionless coupling constants times factors of $\left(16 \pi^{2}\right)^{-n}$, where $n$ is the number of loops. Even in nonperturbative applications [3], such distinctions between trees and loops can be important although less so because of the strong interactions that are involved. It has been observed [6], however, that symmetries associated with the known dynamics, when preserved by the underlying new degrees of freedom, may be used to classify operators arising from the underlying dynamics, irrespective of the particular model.

Given that there is some arbitrariness in the choice of operators, there has been a good deal of recent discussion concerning the "best" choice to make to perform fits to experimental data [7, 8]. A number of people have cited the difficulties deciding among equivalent operators, because some arise in tree-approximation while others may arise only from loop-diagrams [9, 5].

In the past, it has been argued (e.g., in ref. [9]) that, because the equivalence theorem relates some operators arising from loops to operators arising from trees, there is no way to decide a priori which operators to choose. In this paper, we shall discuss the best way to choose among such higher-order operators. The inherent 
ambiguities discussed in ref. [9] can be unraveled in a general way, independent of any particular application. We shall limit our discussion to perturbative application: 2 , where such distinctions are most important, although perhaps this could be extended to other applications. Elsewhere [10], we shall apply this the potential influence of physics beyond the Standard Model (BSM) to the determination of the properties of the observed Higgs boson. This is what inspired the present investigation which, in the end, led to conclusions quite independent of that motivation.

An outline of the paper is as follows: In the next section, we review some features of effective Lagrangians, with particular attention the equivalence theorem. In Appendix A, we discuss some technical complications associated with masses and superrenormalizable couplings. In Section 3, we explain how the equivalence theorem can be used as an equivalence relation to partition the set of operators. In Appendix B, we illustrate these concepts in a simple Yukawa model. In Section 4, we explain that operators may be classified as Potential-Tree-Generated (PTG) operators or Loop-Generated (LG) operators, irrespective of the underlying model or theory, and advocate choosing as basis vectors PTG operators to the extent possible. In Section 5 and Appendix C, we apply this to the SM, and, in Section 6, we revisit our earlier result [6] for triple-gauge-boson couplings (TGB) in this light. Finally, Section 7 summarizes our results.

\section{Some Features of Effective Lagrangians}

We begin by reviewing some of the generic properties of effective field theories. Suppose one has a theory with a Lagrangian of the form

$$
\mathcal{L}=\mathcal{L}^{\prime}\left(\phi_{\ell}\right)+\Delta \mathcal{L}^{\prime}\left(\phi_{\ell}, \phi_{h}\right)
$$

where $\mathcal{L}^{\prime}$ involves "light particles" associated with fields $\phi_{\ell}$ together with additional terms $\Delta \mathcal{L}^{\prime}$ describing the dynamics of some fields $\phi_{h}$ describing interactions among heavy particles and their couplings to light particles. These fields may include both fermions and bosons; no distinction is necessary for present purposes. Correspondingly, one may consider the generating functional (or partition functional)

$$
\mathcal{Z}\left[j_{\ell}, j_{h}\right]=\int \mathcal{D} \phi_{\ell} \mathcal{D} \phi_{h} \exp \left[i \int d x\left\{\mathcal{L}^{\prime}\left(\phi_{\ell}\right)+\Delta \mathcal{L}^{\prime}\left(\phi_{\ell}, \phi_{h}\right)-j_{\ell} \phi_{\ell}-j_{h} \phi_{h}\right\}\right] .
$$

At energy scales below the threshold for heavy particle production, the collision of light particles can only produce light particles, so one may describe their behavior

\footnotetext{
${ }^{2}$ We will assume that we are dealing with relativistic quantum fields in four dimensions.
} 
in terms of light fields alone. The effective action for the theory of light fields is obtained, in path integral language, by "integrating out" the heavy fields, or, in the language of perturbation theory, from Feynman diagrams involving only heavy internal propagators:

$$
\begin{aligned}
\mathcal{Z}\left[j_{\ell}, 0\right] & =\int \mathcal{D} \phi_{\ell} \exp \left[i \int d x\left\{\mathcal{L}_{\text {eff }}\left(\phi_{\ell}\right)-j_{\ell} \phi_{\ell}\right\}\right] \\
\exp \left[i \int d x \mathcal{L}_{\text {eff }}\left(\phi_{\ell}\right)\right] & \equiv \exp \left[i \mathcal{S}_{\text {eff }}\left[\phi_{\ell}\right]\right] \\
& \equiv \exp \left[i \int d x \mathcal{L}^{\prime}\left(\phi_{\ell}\right)\right] \int \mathcal{D} \phi_{h} \exp \left[i \int d x\left\{\Delta \mathcal{L}^{\prime}\left(\phi_{\ell}, \phi_{h}\right)\right\}\right]
\end{aligned}
$$

For the integration over heavy fields in eq. (5)), the light fields $\phi_{\ell}(x)$ play the role of external sources. Perturbatively, the integration represents the sum over all Feynman diagrams having only virtual heavy particles, with all light particles external. In general, the Green's functions thus obtained are nonlocal, but one may expand in inverse powers of the heavy mass scale, which we will call $\Lambda$, to obtain a local effective Lagrangian of the form

$$
\mathcal{L}_{\text {eff }}=\mathcal{L}_{0}\left(\phi_{\ell}\right)+\sum_{i} c_{i} Q_{i}\left(\phi_{\ell}\right)
$$

where $Q_{i}$ are local, Lorentz-invariant and gauge-invariant operators of dimension greater than four with coefficients $c_{i}$ that vanish as inverse powers of the heavy masses and include various positive powers of the coupling constants in $\Delta \mathcal{L}^{\prime}$. For simplicity, we will speak as if there is only a single heavy scale $\Lambda$. The expansion also may involve terms that grow with $\Lambda$, such as $\Lambda^{2} \phi_{\ell}^{2}$ or $\log (\Lambda)\left(\partial \phi_{\ell}\right)^{2}$. Since they necessarily involve operators of dimension four or less, such terms can be absorbed into the coefficients of operators in $\mathcal{L}^{\prime}$ and the wave-function renormalization of the light fields needed to bring the kinetic energy terms in $\mathcal{L}_{0}$ into canonical form 5 . Generically, $\mathcal{L}_{0}$ is identical

\footnotetext{
${ }^{3}$ There are a number of niceties suppressed in this formal summary. One really wants to integrate out the heavy particles and, to avoid having light fields create heavy particles, it is extremely convenient if $\Delta \mathcal{L}^{\prime}$ does not contain quadratic mixing of $\phi_{\ell}$ with $\phi_{h}$, at least not in the kinetic terms if not in the mass terms. This can always be arranged by redefining the fields and by working consistently with the renormalized $\mathcal{L}^{\prime}\left(\phi_{\ell}\right)$, so that $\Delta \mathcal{L}^{\prime}$ contains counterterms that enforce this to arbitrarily high order. Anomalous dimensions and $\beta$-functions may be modified accordingly.

${ }^{4}$ There is no loss of generality here, since one could choose $\Delta \mathcal{L}^{\prime}$ in eq. (11) to be itself an effective field theory. One may simply "integrate out" heavy particles until one arrives at an effective action containing the lightest of the heavy particles.

${ }^{5}$ Thus, the light fields on which $\mathcal{L}_{\text {eff }}$ depend in eq. (6) are not the same as the light fields appearing in eq. (5). These redefinitions have no observable effects and are often implicit in the literature.
} 
in form as $\mathcal{L}^{\prime}$, but with its fields, masses and couplings redefined.

If one knows the underlying theory, eq. (1), then one may calculate the coefficients $c_{i}$ at least to low orders in the loop expansion. At momentum scales below $\Lambda$, the effective Lagrangian eq. (6) may even be a more efficient method of calculation than the original model, eq. (11). One familiar application is to low-energy consequences of the SM, in which one expands in inverse powers of the electroweak scale $v \approx 250 \mathrm{GeV}$ to determine weak interaction effects on quantities such as $g_{\mu}-2$, the anomalous magnetic moment of the muon [11], weak decay amplitudes such as beta-decay of hadrons and nuclei [12], or the electric dipole moment of the electron, neutron, and other particles and atoms or other aspects of aspects of CP-violation [13].

Our current interest however concerns applications of effective field theory to situations in which the correct underlying theory is unknown, as when considering physics beyond the Standard Model (BSM). We may then characterize the entire class of decoupling models by considering operators of successively higher-dimension, starting with dimension-five operators, forming all possible (gauge-invariant) operators $Q_{i}$ of a given dimension composed from the light fields and treating their coefficients $c_{i}$ as additional coupling constants beyond those in $\mathcal{L}_{0}$ to be determined from or constrained by experiment. Since there can be many such operators, it sounds like a daunting task to determine all these new couplings $c_{i}$. Fortunately, there number can be substantially reduced in various ways, but in particular, by use of the equivalence theorem which we will now review.

Operators in quantum field theory satisfy the requirements for complex vector spaces [14]. A set of operators $\left\{Q_{i}\right\}$ will be said to be linearly dependent if a linear combination vanishes. Stated more precisely, suppose a set of constants (possibly depending on coupling constants) $\kappa_{i} \neq 0$ can be found such that

$$
\sum_{i} \kappa_{i} Q_{i}=0
$$

If no such relation exists, then the set of operators $\left\{Q_{i}\right\}$ will be said to be linearly independent. Equality here refers only to perturbation theory, so operators that differ by a total derivative will be considered equal, since their contributions to the action differ only by surface term: 6 . Thus, integration-by-parts (IBP) is allowed, e.g., $(\partial \varphi)^{2}+\varphi \square \varphi \stackrel{I B P}{=}$ 0. So these two operators are linearly dependent. Although not necessary, it is often convenient, especially for renormalization, to choose the operators $\left\{Q_{i}\right\}$ to be irreducible, in the sense that they are contractions of monomials

\footnotetext{
${ }^{6}$ Thus, we ignore any potential, topologically nontrivial terms that may arise.
} 
composed of products of the fields and their covariant derivatives 7 .

The set of all (Lorentz- and gauge-invariant) operators of a given dimension $d$ will be denoted $\mathcal{A}_{d}$. The number of linearly independent operators is called the dimension $\operatorname{dim}\left\{\mathcal{A}_{d}\right\}$ of $\mathcal{A}_{d}$. To reduce the number of operators further requires the equivalence theorem.

\section{Classification by Means of the Equivalence Theorem}

In this section, we will state the equivalence theorem and review without proof some of the properties of equivalence relations, equivalence classes, and quotient spaces. We won't pause to provide an illustration here, but we apply these concepts to a simple Yukawa model in Appendix B. In the next section, we shall marry these ideas with the loop order to select the most phenomenologically useful basis.

Among the operators in $\mathcal{A}_{d}$, certain linear combinations take the form

$$
\sum_{\phi} U_{\phi} \frac{\delta S_{0}}{\delta \phi}+h . c .
$$

where $U_{\phi}$ is some polynomial in the fields and their covariant derivatives. (We implicitly include fermions and vector bosons in the sum.) Here, $S_{0}$ is the action associated with the Lagrangian $\mathcal{L}_{0}$, eq. (6) polynomials $U_{\phi}$ can depend on parameters (masses and couplings) from $\mathcal{L}_{0} . U_{\phi}$ must transform under gauge transformations in such a way that the right-hand side is gauge-invariant. Operators satisfying eq. (8) form a subspace $\mathcal{K}_{d} \subset \mathcal{A}_{d}$. (It is obviously sufficient to find operators of the form $\sum_{\phi} U_{\phi} \delta S_{0} / \delta \phi$, since one can always add its Hermitian conjugate.)

The equivalence theorem may be stated as follows: Operators in $\mathcal{K}_{d}$, i.e., linear combinations of the form of eq. (8), make no contribution to S-matrix element 8 . Thus, operators of this type may be omitted in the construction of the effective Lagrangian, eq. (66). This is a very powerful result that, as we shall see, substantially simplifies $\mathcal{L}_{\text {eff }}$ and reduces the number of operators $Q_{i}$ required.

Two $d$-dimensional operators $Q$ and $Q^{\prime}$ are defined to be equivalent if $\left(Q-Q^{\prime}\right) \in \mathcal{K}_{d}$. In that case, $Q$ and $Q^{\prime}$ give the same contributions to observables. For this reason,

\footnotetext{
${ }^{7}$ Although $\mathcal{L}_{\text {eff }}$ must be Hermitian, it is not always most expedient to make each term in the sum Hermitian; e.g., in the SM, the Yukawa couplings to the Higgs doublet are an example. In such cases, each term is implicitly accompanied by its Hermitian conjugate.

${ }^{8}$ See [15]. See also, e.g., 16] and references therein.
} 
the condition that their difference satisfies eq. (8) is sometimes referred to as the "on-shell" constraint. It can easily be seen that this satisfies the requirements of an equivalence relation [14]. Therefore, every operator $Q$ may be associated with a distinct set $[Q]$ of operators with which it is equivalent $[9$. The set $[Q]$ is called the equivalence class associated with the operator $Q$. The equivalence relation uniquely partitions the original set $\mathcal{A}$ into distinct subsets. The quotient space [14] $\mathcal{M}_{d} \equiv$ $\mathcal{A}_{d} / \mathcal{K}_{d}$ consists of the collection of all such equivalence classes.

The equivalence relation allows one to replace the usual notion of linear independence by inequivalence. Two operators whose difference vanishes "on-shell" can be regarded as identical for the purpose of constructing $\mathcal{L}_{\text {eff }}$, eq. (6). The way to state this formally is to regard the quotient space $\mathcal{M}_{d}$ as a complex vector space, with the null vector being identified with $\left[\mathcal{K}_{d}\right]$. The number of inequivalent classes is the dimension of $\mathcal{M}_{d}$, There is a classic result [14] that $\operatorname{dim}\left\{\mathcal{M}_{d}\right\}=\operatorname{dim}\left\{\mathcal{A}_{d}\right\}-\operatorname{dim}\left\{\mathcal{K}_{d}\right\}$. Choosing one operator from each equivalence class corresponds to selecting a basis set in which to express the higher dimensional operators $Q_{i}$ in $\mathcal{L}_{\text {eff }}$.

We may form the union $\mathcal{A}$ of all such operators $\mathcal{A} \equiv \cup_{d} \mathcal{A}_{d}$, as well as $\mathcal{K} \equiv \cup_{d} \mathcal{K}_{d}$ and $\mathcal{M} \equiv \cup_{d} \mathcal{M}_{d}$. Of course, the dimensions of $\mathcal{A}, \mathcal{K}$, and $\mathcal{M}$ are (denumerably) infinite. Since the elements of the quotient space $\mathcal{M}$ are the unique equivalence classes themselves, there is no ambiguity in selecting a basis for $\mathcal{M}$. Unfortunately, we do not know how to calculate S-matrix elements starting directly from the equivalence classes. It is somewhat analogous to calculations with gauge fields. We know the results are gauge-invariant, but we must choose a gauge in order to perform calculations.

A second, equivalent, way to approach this is to say that an operator $Q$ from a set $\left\{Q_{i}\right\}$ is redundant if it is equal to a finite linear combination of other operators, up to terms that vanish on-shell, i.e.,

$$
Q-\sum_{i} \kappa_{i} Q_{i}=\sum_{\phi} U_{\phi} \frac{\delta S_{0}}{\delta \phi} .
$$

Starting from $\mathcal{A}_{d}$, there will be a minimum number of operators, none of which are redundant. These are the operators that must be included in the effective Lagrangian, eq. (6).

To illustrate, consider simple $\varphi^{4}$ theory

$$
\mathcal{L}_{0}=\frac{1}{2}(\partial \varphi)^{2}-V(\varphi), \quad V(\varphi)=\frac{1}{2} m^{2} \varphi^{2}+\lambda \frac{\varphi^{4}}{4}
$$

\footnotetext{
${ }^{9}$ There is no requirement that the operators under consideration be monomials.
} 
The EoM is $\delta \mathcal{S}_{0} / \delta \varphi=-\partial^{2} \varphi-V^{\prime}(\varphi)$. Considered as a model of low-energy physics, $V(\varphi)$ has a discrete $Z_{2}$ symmetry $\varphi \rightarrow-\varphi$. We wish to consider possible extensions of the theory as in eq. (1), where $\mathcal{L}^{\prime}$ has the same form as $\mathcal{L}_{0}$. Both are expressed in terms of renormalized fields, so that, implicitly, $\Delta \mathcal{L}^{\prime}$ contains appropriate counterterms. In general, we must assume that $\Delta \mathcal{L}^{\prime}$ respects this $Z_{2}$ symmetry since otherwise, the effective Lagrangian, eq. (6), would contain a term of the form $A \phi^{3}$, where $A$ has dimensions of mass. This would be required for renormalizability of eq. (66). Thus, the higher dimensional operators in eq. (6) would involve only even powers of $\phi$, so the lowest order terms would have dimension six.

This is already a non-trivial result, since it means that the first corrections must be of $O\left(1 / \Lambda^{2}\right)$ rather than $O(1 / \Lambda)$. Considering dimension-six operators, we have $\left\{\varphi^{6}\right.$, $\left.\varphi^{2}(\partial \varphi)^{2},\left(\partial^{2} \varphi\right)^{2}\right\}$. Of these three, only one is inequivalent, which we will take to be $\varphi^{6}$. To see this, note that

$$
\left(\partial^{2} \varphi\right)^{2}-V^{\prime}(\varphi)^{2}=-\left(\partial^{2} \varphi-V^{\prime}(\varphi)\right) \frac{\delta S_{0}}{\delta \varphi}
$$

Now $V^{\prime}(\varphi)^{2}=\left(m^{2} \varphi+\lambda \varphi^{3}\right)^{2}=m^{4} \varphi^{2}+2 m^{2} \lambda \varphi^{4}+\lambda^{2} \varphi^{6}$. This shows that the operator $\left(\partial^{2} \varphi\right)^{2}$ is not inequivalent to the operators $\left\{\varphi^{6}, \varphi^{4}, \varphi^{2}\right\}$.

The occurrence of lower-dimensional operators $\left\{\varphi^{2}, \varphi^{4}\right\}$ in this equivalence forces us into a slightly technical digression which however has consequences for construction of all effective field theories. Obviously the occurrence of operators of lower dimension in such relations is associated with light masses, here $m^{2}$, or with super-renormalizable couplings. This complication may be dealt with in several different ways, as elaborated in Appendix A. The upshot is that, for the purpose of determining inequivalence of operators of a given dimension, one may simply ignore lower-dimensional operators arising from application of the EoM.

Returning from this digression to classifying dimension-six operators, we have shown that $\left(\partial^{2} \varphi\right)^{2}$ is equivalent to $\varphi^{6}$. The same is true for $\varphi^{2}(\partial \varphi)^{2}$. To see this, first note that, after IBP, this operator is identical to $-\varphi \partial_{\mu}\left(\varphi^{2} \partial^{\mu} \varphi\right)=-\varphi^{3} \partial^{2} \varphi-2 \varphi^{2}(\partial \varphi)^{2}$, so that $\varphi^{2}(\partial \varphi)^{2} \stackrel{I B P}{=}-\varphi^{3} \partial^{2} \varphi / 3$. Then we may use the EoM to replace $\partial^{2} \varphi$,

$$
\varphi^{3} \partial^{2} \varphi+\varphi^{3} V^{\prime}(\varphi)=-\varphi^{3} \frac{\delta S_{0}}{\delta \varphi} .
$$

Ignoring masses, the second term is simply $\lambda \varphi^{6}$. Therefore, the only inequivalent correction of dimension six to the effective Lagrangian can be taken to be $c_{\varphi} \varphi^{6}$, as was claimed. 
This simple example illustrates that one can continue this process of eliminating redundant operators until one reaches a certain minimum number of a given dimension. We call this set a basis; note that the linear (in $Q$ ) nature of the relation eq. (9) implies that the number of elements in a basis is invariant. It also suggests that, generally, it is easiest to use the EoM to remove operators having multiple (covariant) derivatives. This strategy has other benefits that will be explained subsequently.

Although the choice of basis operators for $\mathcal{M}_{d}$ is arbitrary, for present purposes, we will want to select them with reference to their order in the loop expansion, as will be discussed in the next section.

\section{Trees, Loops, \& Choice of Basis}

Returning to eq. (11), let us consider all possible extensions $\Delta \mathcal{L}^{\prime}\left(\phi_{\ell}, \phi_{h}\right)$ of a theory described by $\mathcal{L}^{\prime}\left(\phi_{\ell}\right)$. We wish to consider models having particles heavy with respect to the particles described by $\mathcal{L}^{\prime}$. If not strongly interacting, these too are expected to take the form of effective field theories, which implies that the most relevant terms in $\Delta \mathcal{L}^{\prime}$ will have dimensions four or less. We will argue that the generic form of such theories can be delineated without further assumptions and, correspondingly, the leading observable corrections to $\mathcal{L}^{\prime}$ discussed without reference to a specific underlying theory. Further, there is a way of selecting basis sets $\left\{Q_{i}\right\}$ for eq. (6) that, while not unique, are optimal, in a sense described below.

Models will have fields representing possible scalars, fermions, and vectors 10 . These must be consistent with the gauge symmetries associated with $\mathcal{L}^{\prime}$, and possibly with discrete or global symmetries as well. The interactions of the heavy fields with the light ones in $\mathcal{L}^{\prime}$ can only take the form of scalar self-interactions, Yukawa-like interactions of scalars and fermions, and interactions with vectors as dictated by the gauge symmetries. As a first approximation, the most important of these will usually be tree diagrams, so all the corresponding vertices must contain at least one light field and one heavy field. Using this, one may write down all possible vertices consistent with the symmetries of $\mathcal{L}^{\prime}$ and draw all possible tree diagrams having external light particles and internal heavy particles. As an illustration, this was done for the SM in ref. [6]; (cf. Fig. 3 in this reference.)

From such diagrams, one may work out which operators $Q_{i}$ of a given dimension $d$ may arise in the effective Lagrangian, eq. (6) , from tree diagram: 11 . These operators

\footnotetext{
${ }^{10}$ While gravity could be included, we assume it is irrelevant for the applications we have in mind, such as to LHC data.

${ }^{11}$ Depending on the context, one may instead consider restricted extensions of the theory, such as
} 
form a subset of the complete set of operators of a given dimension $d$, and the vector space spanned by them form a subspace of the space $\mathcal{A}_{d}$ of all operators of dimension $d$. This subspace will be referred to as the Potential-Tree-Generated (PTG) operators of dimension $d$ and denoted as $\mathcal{A}_{d}^{P T G}$. In practice, one will often only be interested in the corrections of lowest order, operators of dimension five or six, although there are exception: 12 .

Whether an operator is generated by a tree graph or only via loops depends on the details of the underlying theory. The set $\mathcal{A}_{d}^{P T G}$ consists of those operators that are generated at tree-level in at least one extension of the low-energy theory. In the case of the SM as the low-energy theory, which describes nature well, there is no guarantee that the BSM physics will in fact generate any of these at tree level, hence our use of the qualifier "potential".

How should one go about selecting a basis set for $\mathcal{A}$ ? In order to deal with a finite number of operators, we shall focus on PTG operators of a fixed dimension $d$. Defining $\left[\mathcal{A}_{d}^{P T G}\right]$ to be the set of equivalence classes associated with all these operators, one may consider its intersection with the quotient space $\mathcal{M}_{d} \cap\left[\mathcal{A}_{d}^{P T G}\right]$. For any equivalence class for which the intersection is non-empty, it behooves us to select a basis operator for that class from among operators in the intersection, because the PTG operators generically have larger coefficients than the LG operators 13 . Stated more physically, even though an equivalence class may contain both operators from trees and from loops in the underlying theory, without knowing the correct BSM dynamics, we ought to allow that the coefficients are as large as potentially possible, since there are types of BSM physics that do produce these operators at tree level. Put yet another way, if a LG operator were selected as the representative of this equivalence class, experimental evidence for its presence may be misinterpreted as being associated with a much lower threshold of new physics than if it were a PTG operator. We call the equivalence classes spanned by these classes $\mathcal{M}_{d}^{P T G}$. If there remain any equivalence classes for which the intersection is empty, then it is fair to call these classes loop-generated (LG) $\mathcal{M}_{d}^{L G}$, since they cannot arise from a tree graph in any extension of $\mathcal{L}^{\prime}$. Phenomenologically, this implies that the coefficients $c_{i}^{P T G}$ of the dimension-d basis operators of $\mathcal{M}_{d}^{P T G}$ take the form $f_{i} / \Lambda^{d-4}$, with dimensionless

those having only additional fermions or those having only a single additional heavy vector boson or those respecting baryon-number conservation. The discussion here may be applied to such restricted cases as well.

${ }^{12}$ See, e.g., 19 and references therein.

${ }^{13} \mathrm{We}$ are not the first to suggest this criterion. See [8]. 
constants $f_{i} \sim O(1)$ in all models where these operators are in fact tree-generated 14 . On the other hand, for operators from $\mathcal{M}_{d}^{L G}$, the coefficients may be assumed to be $f_{i} \sim O\left(1 / 16 \pi^{2}\right)$ for any underlying theory.

For a simple example of how these concepts can be applied in practice, we again refer to Appendix B, where we classify operators as PTG or LG and discuss the choice of basis operators for a simple Yukawa model. We want to move on to discuss the SM, but there are a couple of caveats to keep in mind: (1) The equivalence of two operators is a property of the low-energy theory alone. It is possible for the underlying theory to generate $Q$ and not $Q^{\prime}$, even if the two operators are equivalent. (2) It is possible for LG and PTG operators to be equivalent. This last point might appear odd, given the difference in the operator coefficients. To understand this better it is worth emphasizing that what the equivalence theorem says is that the observable effects of one operator can be mimicked by those of another one, it does not provide any insights on the structure of the underlying physics. If $Q_{\mathrm{PTG}}, Q_{\mathrm{LG}}$ are equivalent PTG and LG operators and are in fact generated by some heavy dynamics such that the first appears at tree-level, the equivalence theorem does not imply that the effects of $Q_{\mathrm{LG}}$ are promoted to tree-level status. Instead it says that explicit calculation will show that the particles responsible for generating $Q_{\mathrm{PTG}}$ will provide deviations from the SM at tree-level, while those that responsible for $Q_{\mathrm{LG}}$ will generate small additive corrections as long as the typical energies are below $\Lambda$. Above this scale, the PTG diagrams will exhibit resonances (in the appropriate channel) while LG graphs will contain the effects associated with unitarity cuts - of course, in this regime the effective Lagrangian approach will no longer be applicable.

Usually, one would be satisfied seeking effects from the lowest dimensional operators that may occur, but with some effort, one could do even better. From these classes $\mathcal{M}_{d}^{L G}$, one could identify all operators that may arise at one-loop order in some underlying theory. If these did not exhaust all these equivalence classes, it would make sense to define subspaces $\mathcal{M}_{d}^{1 L G}$, with others arising in higher order. Phenomenologically, this is only interesting to do if one could measure some S-matrix elements that could not arise from an underlying theory until two-loops or higher. This situation does in fact occur, for example, in the calculation of electric dipole moments [13] in certain models of CP-violation.

The different choices of basis operators will give different Green's functions in

\footnotetext{
${ }^{14}$ As mentioned earlier, it may be that a naturalness requirement or other constraint could influence expectations or interpretations. E.g., if, because of an enhanced chiral symmetry, each SM fermion mass vanishes in the limit of vanishing Yukawa couplings, then $f_{i}$ for operators contributing to corrections to Yukawa couplings must be proportional to a power of the SM fermion masses.
} 
general, even though each choice yields the same S-matrix elements. One must be careful not to omit any basis operators without good reason, since their contributions to Green's functions can be very different. An incomplete basis set may lead to spurious relations among observables.

\section{SM Operators of Dimension Six}

To put the preceding prescription into practice, we have classified all the operators of dimension six for the SM, assuming the absence of right-handed neutrinos and baryon- and lepton-conservation. (If these are violated, we assume it is at a much higher scale, as suggested by limits on proton decay and on searches for leptonviolation [18].) The application of the equivalence theorem to the SM was originally performed in a now-classic paper by Buchmüller \& Wyler [4] (BW.) It has been shown recently by Grzadkowski et al. [5] (GIMR) that the BW "basis" in fact still contained redundant operators. We will use the GIMR basis, which, for easy reference, is reproduced in part in Appendix $\mathrm{C}$, omitting the baryon-violating operators.

The equivalence relations are summarized in Appendix D. If one wishes to understand the implications for the equivalence classes, one may simply replace each operator $Q$ by its corresponding equivalence class $[Q]$, and replace equivalence, $\sim$, by equality, $=$, as was illustrated in Appendix B.

To emphasize a point made in the previous section, we note that eq. (D.7) is an example of an equivalence relation involving both PTG and LG operators. One can show that $\left(D^{\mu} W_{\mu \nu}^{I}\right)^{2}$ is a LG operator, however every operator on the right hand side of that equivalence relation is a PTG operator. This also illustrates that a particular sum of PTG operators can be equivalent to a LG operator 15 .

For our purposes, the specific formulae are less important than their implications for the choice of basis operators. We have analyzed the GIMR basis operators and determined which are PTG and which are LG. Their basis can be classified as follows:

$$
\begin{aligned}
\text { PTG types : } & \varphi^{6}, \varphi^{4} D^{2}, \psi^{2} \varphi^{3}, \psi^{2} \varphi^{2} D, \psi^{4} . \\
\text { LG types : } & X^{3}, X^{2} \varphi^{2}, \psi^{2} X \varphi .
\end{aligned}
$$

By definition, no basis operator can be written as a linear combination of other basis operators. Even though certain linear combinations of PTG operators can

\footnotetext{
${ }^{15}$ Although this has been asserted earlier in [17], the example given there is not correct.

${ }^{16}$ The type denoted $\psi^{4}$ includes all four-fermion operators. It turns out all are PTG, regardless of their Lorentz or chiral structure.
} 
be equivalent to a LG operator, the basis chosen in [5] is optimal in the following sense: none of their $L G$ basis operators requires replacement by a PTG operator, i.e., the equivalence class of each LG basis operator contains no PTG operators. To establish this, one must examine each equivalence relation in Appendix D in which a given LG operator occurs to determine whether there are any PTG operators in that class. The proof is by exhaustion; one need only examine each equivalence relation in Appendix D in which a given LG operator occurs and verify that there are no PTG basis operators in that class. It seems the strategy of eliminating as many (covariant) derivatives as possible has this unanticipated benefit.

\section{Triple Vector Boson Corrections}

As an application of the preceding, we return to the topic of corrections to triplegauge-boson (TGB) couplings from BSM physics. In earlier work [6] , we showed that corrections to triple vector boson couplings do not arise in tree approximation in any extension of the SM. In so doing, we compared operators arising from all possible tree diagrams with the basis operators elaborated by Büchmuller and Wyler (BW) [4]. There are two things we must do to update that analysis. First, as mentioned earlier, it has been shown recently [5] that the basis of [4] contains redundant operators, so we should determine whether this alters any of the conclusions of [6] or [17]. Second, according to the discussion above, it is not sufficient to complete the analysis using one particular choice of basis operators, since a LG operator may be equivalent to a linear combination of PTG operators. Actually, this second issue is the easier, since it was addressed in the preceding section. Once we narrow down to the basis choice in [17], then following the argument in section 4, it is not hard to see that none of their LG operators is equivalent to a linear combination of PTG operators.

Returning to the first issue, the basis operators in [4] that are redundant have been reviewed in Section 3 of [5], to which we refer for further details. Of the BW operators that affect the TGB couplings, $\mathcal{O}_{\phi}^{(1)} \equiv\left(\phi^{\dagger} \phi\right)\left(D_{\mu} \phi\right)^{\dagger} D^{\mu} \phi$ is redundant, but it is also a PTG operator that is equivalent to a linear combination of other PTG operators, so that is not a problem. Similarly, the elimination of some four-fermion operators by Fierz transformations is irrelevant to the TGB corrections. None of the redundant operators identified in [5] having covariant derivatives on fermion fields can contribute to the TGB couplings. Thus, dropping the redundant operators from the BW "basis" has no effect on the analysis of the TGB couplings in [6].

Therefore, none of the more recent developments vitiates the conclusions in [6] . In any extension of the SM, corrections to the TGB couplings are suppressed by at least 
one-loop order. Thus, assuming that the BSM dynamics is decoupling, the magnitude of the coefficients should only be a few parts per thousand of the SM gauge coupling.

Note that if a process is not PTG, then whether the corresponding dimension-six operator should be retained or ignored depends not only on the on the process but also whether the process is tree- or loop-generated in the SM. If it is SM tree-generated (SMTG,) a BSM LG is probably going to be too small to observe, but if the process is SM loop-generated (SMLG), then a BSM loop operator (BSMLG) is only suppressed by a factor of $\epsilon=v^{2} / \Lambda^{2}$, just like a process that is SMTG having PTG operators from BSM.

It is ironic in this context that the dominant production mechanism for the production of the Higgs boson at LHC is gluon fusion (which is a SMLG amplitude). Similarly, one of the most easily identified decay channels is $H \rightarrow \gamma \gamma$, which is SMLG as well. We shall discuss this further elsewhere [10].

Finally, note that the same is true of quartic vector boson couplings, viz. the only dimension-six operators contributing to these are LG. Indeed, since they are associated with the same operators as modifications of the triple-vector-boson couplings, the magnitude of the two are correlated.

Of course, for both triple and quartic couplings, it is possible that other HDO, such as dimension-eight PTG operators, could be more important than dimensionsix LG operators, depending on the scale of BSM physics. The exploration of these implications can await experimental evidence for any deviations from the SM.

\section{Conclusion}

The purpose of this paper was to discuss how one might use the equivalence relation not simply to establish the inequivalent operators but also to determine the equivalence classes of operators. This is similar to the demonstration of independence in [5], but we needed to make the equivalence classes explicit in order to ascertain which among them contain PTG operators; which, only LG operators; and which, both types. We advocated choosing a basis with the maximum number of PTG operators so that we most easily and reliably interpret fits to data. Even if no new physics is indicated by experimental results, this strategy allows for a figure of merit to be assigned and to infer the likely scale $\Lambda$ above which we are ignorant. With further restrictions on the BSM model, such as supposing certain other global symmetries obtain, one may draw other inferences.

We applied this to a classification of the dimension-six basis operators of the SM, concluding that none of the LG basis operators in [5] requires replacement by a PTG operator. We also revisited the triple-gauge boson (TGB) couplings in the present 
light, reaffirming our earlier results [6] that corrections to the TGB couplings do not arise at tree level.

In a certain sense, this classification scheme is renormalization group invariant, as can be deduced from earlier work [20]. Notice first that the beta functions for the coefficients of the HDO's can be determined from Feynman rules involving only the basis set. Although redundant operators may arise in this or another equivalence class, one may use the EoM to rewrite any such operator in terms of a sum of basis operators. Under renormalization, operator mixing can occur with, for example, a PTG vertex contributing to the running of the coupling constant (coefficient) of a LG operator. However, this does not invalidate our conclusions. Consider a dimensionsix LG operator. If we simply connect two external legs, we get a renormalization of a dimension-four operator, which we have agreed to absorb into the renormalized couplings of the SM. So we must insert at least one SM vertex to obtain another dimension-six operator. Thus, the evolution of the coupling constant multiplying a LG operator can involve a PTG vertex, giving a correction equal to the PTG vertex times a product of SM couplings, times at least one loop-factor of $1 / 16 \pi^{2}$. This is at most of the same order as the original LG coupling and so does not upset the relative magnitude of the coefficients of PTG and LG operators.

It would be helpful if analytical methods could be found for identifying PTG and LG operators for any effective field theory. At present, the only method we know is to delineate all vertices and to analyze all potential tree graphs to determine which give rise to PTG operators.

There are obviously many opportunities to analyze data using these new insights. In a related paper [10], we apply this scheme to the production and decay of the SM Higgs boson.

\section{Acknowledgments}

We would like to thank the authors of ref. [5] for permission to use their tables. MBE wishes to thank J. R. Espinosa for discussions. The research of one of us (MBE) was supported in part by the National Science Foundation under Grant No. NSF PHY11-25915.

\section{Appendix A. Equivalence and Operators of Lower Dimension}

The purpose of this appendix is to argue that lower dimensional operators that occur in equivalence relations may be ignored because they simply provide renormalizations of couplings already extant in lower orders of the effective field theory. Such 
operators arise with coefficients proportional to powers of superrenormalizable couplings in $\mathcal{L}_{0}$ or to masses of light fermions and bosons. This complication may be dealt with in several different ways. One may simply absorb such terms into the coefficients of the lower dimensional operators in $\mathcal{L}_{\text {eff }}$, or, perhaps even more simply, one may assume that the lower dimensional operators are expressed in terms of renormalized fields and couplings, so that in addition to the terms $\sum c_{i} Q_{i}$, the interaction contains counterterms that cancel any corrections to operators already appearing in $\mathcal{L}_{\text {eff }}$. Referring to the example at the end of section 2, we can say, e.g., that $\left\{\left(\partial^{2} \varphi\right)^{2}, \varphi^{6}\right\}$ are not inequivalent dimension-six operators, with the understanding that the equivalence may tacitly include operators of lower dimension as wel 17 .

The easiest way to deal with this complication in general is to temporarily set to zero all masses or superrenormalizable couplings in $\mathcal{L}_{0}$. Then complete the classification of higher dimensional operators with the understanding that, when these parameters are restored, there will arise operators of lower dimension to be canceled by appropriate counterterms. This is the prescription that we will always follow, so that it is as if operators of a given dimension do not mix with operators of lower dimension under this equivalence relation. (The only caveat is that one must be sure that all such lower-dimensional operators actually have been included.)

This technique is in harmony with mass-independent methods of renormalization, such as dimensional regularization and minimal subtraction, the standard method for treating gauge theories. In this way, renormalization of effective field theories remains consistent with multiplicative renormalization. Operators only mix with other operators of the same dimension.

\section{Appendix B. Simple Yukawa Model}

In this appendix, we shall apply the formalism to determine the equivalence classes for a simple model. Consider a Lagrangian with a scalar field $\phi$ and a fermion $\psi$ :

$$
\mathcal{L}=\frac{1}{2}(\partial \phi)^{2}-\frac{1}{2} m^{2} \phi^{2}-\frac{\lambda}{4} \phi^{4}+\bar{\psi}(i \not \partial-u \phi) \psi
$$

this has a $\mathrm{Z}_{2}$ chiral symmetry $\phi \rightarrow-\phi, \psi \rightarrow \gamma_{5} \psi$. This symmetry rules out a Dirac mass $m_{\psi} \bar{\psi} \psi$. This Lagrangian is also parity invariant, $\psi \rightarrow \gamma^{0} \psi, \phi \rightarrow+\phi$.

\footnotetext{
${ }^{17}$ One must keep in mind that this prescription does not take into account quantities such as $m^{2} / \Lambda^{2}$, whose size must be compared to those of various renormalized couplings to which they may contribute. This is similar to the issue of naturalness, which transcends renormalizability.
} 
The equation of motion are $\mathcal{E}_{\phi}=0, \mathcal{E}_{\psi}=0$ where

$$
\begin{aligned}
\mathcal{E}_{\phi} & \equiv \square \phi+\lambda \phi^{3}+u \bar{\psi} \psi \\
\mathcal{E}_{\psi} & \equiv(i \not \partial-u \phi) \psi
\end{aligned}
$$

Considering extensions of this theory, what properties shall we require? We will assume that it respects the $\mathrm{Z}_{2}$ chiral symmetry; otherwise, we would expect the low energy theory to have a Dirac mass. Whether it must conserve parity is less clear, especially given experience with the differences between electromagnetism and the electroweak theory. For simplicity, we will assume parity is conserved as well 18 .

Because of the $\mathrm{Z}_{2}$ chiral symmetry, there are no dimension-five operators. It turns out that there are then seven types of dimension-six operator 19 :

$$
\begin{aligned}
& \text { 1. } \phi^{6}: Q_{1} \equiv \phi^{6} \\
& \text { 2. } \phi^{4} \partial^{2}: \quad Q_{2} \equiv \phi^{3} \square \phi \\
& \text { 3. } \phi^{3} \psi^{2}: \quad Q_{3} \equiv \phi^{3} \bar{\psi} \psi \\
& \text { 4. } \phi^{2} \partial^{4}: \quad Q_{4} \equiv(\square \phi)^{2} \\
& \text { 5. } \phi^{2} \psi^{2} \partial: Q_{5} \equiv \phi^{2} \bar{\psi} i \not \partial \psi \text {, and } Q_{5}^{\dagger} \\
& \text { 6. } \phi \psi^{2} \partial^{2}: Q_{6} \equiv(\square \phi)(\bar{\psi} \psi) ; Q_{7} \equiv(\overline{i \not \partial \psi} i \not \partial \psi) \phi ; \quad(L G) \\
& Q_{8} \equiv \phi(\bar{\psi} \square \psi) \text {, and } Q_{8}^{\dagger}
\end{aligned}
$$

Finally, we have operators of the form $\psi^{4}:\left(\bar{\psi} \Gamma_{a} \psi\right)\left(\bar{\psi} \Gamma^{a} \psi\right)$ with $\Gamma_{a} \equiv \mathbb{1}, \gamma_{5}, \gamma_{\mu}, \gamma_{5} \gamma_{\mu}$, $\sigma_{\mu \nu}$, that we refer to as $S, P, V, A, T$. The Fierz relations imply

$$
\begin{aligned}
& 2 T=-6 S+T-6 P \Rightarrow T=-6(S+P) \\
& 2 V=-2 S+V+A+2 P \Rightarrow A=V+2 S-2 P,
\end{aligned}
$$

so there are only three independent four-fermion operators, which we may choose as

$$
\text { 7. } Q_{9} \equiv S(P T G), \quad Q_{9}^{\prime} \equiv P(P T G), \quad Q_{10} \equiv V(P T G) .
$$

The equivalence relation is defined as

$$
Q \sim Q^{\prime} \Leftrightarrow Q-Q^{\prime}=0 \text { when } \mathcal{E}_{\phi}=\mathcal{E}_{\psi}=0 .
$$

Then

$$
\begin{aligned}
& Q_{2} \sim-\lambda Q_{1}-u Q_{3} \quad Q_{4} \sim-\lambda Q_{2}-u Q_{6} \\
& Q_{5} \sim u Q_{3} \quad Q_{6} \sim-\lambda Q_{3}-u Q_{9} \\
& Q_{7} \sim u^{2} Q_{3} \quad Q_{8} \sim-u^{2} Q_{3}
\end{aligned}
$$

\footnotetext{
${ }^{18}$ If we were describing a physical system rather than a simple model, these would of course be testable assumptions.

${ }^{19}$ Ignore their designations as (PTG) or (LG) for now.
} 
From these, it follows that $Q_{4} \sim \lambda^{2} Q_{1}+2 \lambda u Q_{3}+u^{2} Q_{9}$, so that is not an independent relation. There are also the operators $Q_{9}^{\prime}$ and $Q_{10}$ which do not appear in these relations, and so are inequivalent.

For the quotient space, we can simply replace in the preceding equations $Q_{i} \rightarrow\left[Q_{i}\right]$ and $\sim \rightarrow=$ :

$$
\begin{array}{ll}
{\left[Q_{2}\right]=-\lambda\left[Q_{1}\right]-u\left[Q_{3}\right]} & {\left[Q_{4}\right]=-\lambda\left[Q_{2}\right]-u\left[Q_{6}\right]} \\
{\left[Q_{5}\right]=u\left[Q_{3}\right]} & {\left[Q_{6}\right]=-\lambda\left[Q_{3}\right]-u\left[Q_{9}\right]} \\
{\left[Q_{7}\right]=u^{2}\left[Q_{3}\right]} & {\left[Q_{8}\right]=-u^{2}\left[Q_{3}\right]}
\end{array}
$$

These six constraints among nine equivalence classes suggest that we have at most 3 distinct equivalence classes among them. With the benefit of hindsight, we may choose them to be, e.g., $\left[Q_{1}\right],\left[Q_{3}\right]$, and $\left[Q_{9}\right]$. The other 6 therefore are related; indeed,

$$
\begin{array}{ll}
{\left[Q_{2}\right]=-\lambda\left[Q_{1}\right]-u\left[Q_{3}\right]} & {\left[Q_{4}\right]=\lambda^{2}\left[Q_{1}\right]+2 \lambda u\left[Q_{3}\right]+u^{2}\left[Q_{9}\right]} \\
{\left[Q_{5}\right]=u\left[Q_{3}\right]} & {\left[Q_{6}\right]=-\lambda\left[Q_{3}\right]-u\left[Q_{9}\right]} \\
{\left[Q_{7}\right]=u^{2}\left[Q_{3}\right]} & {\left[Q_{8}\right]=-u^{2}\left[Q_{3}\right]}
\end{array}
$$

In addition, there are the two single element equivalence classes $\left[Q_{9}^{\prime}\right]$ and $\left[Q_{10}\right]$, which are inequivalent to others. Thus, there are 5 independent equivalence classes, so there must be 5 independent basis operators.

Which five basis operators shall we choose? Obviously, the equivalence relations have established that $Q_{9}^{\prime}$ and $Q_{10}$ must be chosen as basis operators, since their associated equivalence classes contain only a single element. For the remaining three equivalence classes, according to our general approach, we want to consider the relations among equivalence classes and, whenever possible, choose PTG operators.

Suppose we have an extension of this model described as in eq. (11) whose heavy particles generate (some of) the effective operators $Q_{i}$. We will assume that the kinetic energy terms for $\phi_{\ell}$ and $\phi_{h}$, which implicitly include fermions and gauge fields, and that the quadratic mass matrices contain no mixing term $20 \propto \phi_{\ell} \phi_{h}$. In the absence of a quadratic mixing, the vertex structure of possible extensions is like that of the SM, which we have already discussed [6]. An examination of Fig. 3 in this reference leads to the classification of the eleven dimension-six operator 21 as (PTG) or (LG), as we have denoted in the equations above. There are seven PTG operators and four LG operators. The relations eq. (B.11) show that the equivalence classes associated

\footnotetext{
${ }^{20}$ If that is not the case, one can redefine the fields by performing a global orthogonal (if real) or unitary (if complex) transformation of the fields to bring it to this form. Of course, one may have to adopt a renormalization procedure to sustain the absence of such terms.

${ }^{21}$ The Hermitian conjugates, $Q_{5}^{\dagger}$ and $Q_{8}^{\dagger}$, are not counted separately.
} 
with all four LG operators, $\left[Q_{4}\right],\left[Q_{6}\right],\left[Q_{7}\right],\left[Q_{8}\right]$, may be expressed in terms of classes associated with PTG operators. So we want to choose a basis from among the PTG operators, viz., $Q_{1}, Q_{3}$, and $Q_{9}$. The remaining two basis operators, $Q_{9}^{\prime}$ and $Q_{10}$, are also PTG operators. Therefore, we can choose a complete set of 5 basis operators that are all PTG.

If this model were actually testable experimentally and precise measurements were carried out that determined or placed limits on the five independent coupling constants $c_{i}$ associated with these operators, then one may be able to infer some hints about the underlying extended theory. For example, if there were evidence for the presence of the operator $Q_{10}$, but, with the same precision, no evidence for $Q_{9}$ or $Q_{9}^{\prime}$, one would be strongly motivated to search for a heavy vector boson. Assuming its gauge couplings, parameterized by $f_{10}$, were $O(1)$, we could infer an approximate upper limit on its mass. 


\section{Appendix C. Dimension-Six Basis Operators for the SM22.}

\begin{tabular}{|c|c|c|c|c|c|}
\hline \multicolumn{2}{|r|}{$X^{3}(\mathrm{LG})$} & \multicolumn{2}{|c|}{$\varphi^{6}$ and $\varphi^{4} D^{2}$ (PTG) } & \multicolumn{2}{|r|}{$\psi^{2} \varphi^{3}$ (PTG) } \\
\hline$Q_{G}$ & $f^{A B C} G_{\mu}^{A \nu} G_{\nu}^{B \rho} G_{\rho}^{C \mu}$ & $Q_{\varphi}$ & $\left(\varphi^{\dagger} \varphi\right)^{3}$ & $Q_{e \varphi}$ & $\left(\varphi^{\dagger} \varphi\right)\left(\bar{l}_{p} e_{r} \varphi\right)$ \\
\hline$Q_{\widetilde{G}}$ & $f^{A B C} \widetilde{G}_{\mu}^{A \nu} G_{\nu}^{B \rho} G_{\rho}^{C \mu}$ & $Q_{\varphi \square}$ & $\left(\varphi^{\dagger} \varphi\right) \square\left(\varphi^{\dagger} \varphi\right)$ & $Q_{u \varphi}$ & $\left(\varphi^{\dagger} \varphi\right)\left(\bar{q}_{p} u_{r} \widetilde{\varphi}\right)$ \\
\hline$Q_{W}$ & $\varepsilon^{I J K} W_{\mu}^{I \nu} W_{\nu}^{J \rho} W_{\rho}^{K \mu}$ & $Q_{\varphi D}$ & $\left(\varphi^{\dagger} D^{\mu} \varphi\right)^{\star}\left(\varphi^{\dagger} D_{\mu} \varphi\right)$ & $Q_{d \varphi}$ & $\left(\varphi^{\dagger} \varphi\right)\left(\bar{q}_{p} d_{r} \varphi\right)$ \\
\hline$Q_{\widetilde{W}}$ & $\varepsilon^{I J K} \widetilde{W_{\mu}^{I \nu}} W_{\nu}^{J \rho} W_{\rho}^{K \mu}$ & & & & \\
\hline \multicolumn{2}{|r|}{$X^{2} \varphi^{2}(\mathrm{LG})$} & \multicolumn{2}{|r|}{$\psi^{2} X \varphi(\mathbf{L G})$} & \multicolumn{2}{|r|}{$\psi^{2} \varphi^{2} D$ (PTG) } \\
\hline$Q_{\varphi G}$ & $\varphi^{\dagger} \varphi G_{\mu \nu}^{A} G^{A \mu \nu}$ & $Q_{e W}$ & $\left(\bar{l}_{p} \sigma^{\mu \nu} e_{r}\right) \tau^{I} \varphi W_{\mu \nu}^{I}$ & $Q_{\varphi l}^{(1)}$ & $\left(\varphi^{\dagger} i \stackrel{\leftrightarrow}{D}_{\mu} \varphi\right)\left(\bar{l}_{p} \gamma^{\mu} l_{r}\right)$ \\
\hline$Q_{\varphi \widetilde{G}}$ & $\varphi^{\dagger} \varphi \widetilde{G}_{\mu \nu}^{A} G^{A \mu \nu}$ & $Q_{e B}$ & $\left(\bar{l}_{p} \sigma^{\mu \nu} e_{r}\right) \varphi B_{\mu \nu}$ & $Q_{\varphi l}^{(3)}$ & $\left(\varphi^{\dagger} i \stackrel{\leftrightarrow}{D}{ }_{\mu}^{I} \varphi\right)\left(\bar{l}_{p} \tau^{I} \gamma^{\mu} l_{r}\right)$ \\
\hline$Q_{\varphi W}$ & $\varphi^{\dagger} \varphi W_{\mu \nu}^{I} W^{I \mu \nu}$ & $Q_{u G}$ & $\left(\bar{q}_{p} \sigma^{\mu \nu} T^{A} u_{r}\right) \widetilde{\varphi} G_{\mu \nu}^{A}$ & $Q_{\varphi e}$ & $\left(\varphi^{\dagger} i \stackrel{\leftrightarrow}{D}_{\mu} \varphi\right)\left(\bar{e}_{p} \gamma^{\mu} e_{r}\right)$ \\
\hline$Q_{\varphi \widetilde{W}}$ & $\varphi^{\dagger} \varphi \widetilde{W}_{\mu \nu}^{I} W^{I \mu \nu}$ & $Q_{u W}$ & $\left(\bar{q}_{p} \sigma^{\mu \nu} u_{r}\right) \tau^{I} \widetilde{\varphi} W_{\mu \nu}^{I}$ & $Q_{\varphi q}^{(1)}$ & $\left(\varphi^{\dagger} i \stackrel{\leftrightarrow}{D}_{\mu} \varphi\right)\left(\bar{q}_{p} \gamma^{\mu} q_{r}\right)$ \\
\hline$Q_{\varphi B}$ & $\varphi^{\dagger} \varphi B_{\mu \nu} B^{\mu \nu}$ & $Q_{u B}$ & $\left(\bar{q}_{p} \sigma^{\mu \nu} u_{r}\right) \widetilde{\varphi} B_{\mu \nu}$ & $Q_{\varphi q}^{(3)}$ & $\left(\varphi^{\dagger} i \stackrel{\leftrightarrow}{D} \stackrel{\leftrightarrow}{I}^{\prime} \varphi\right)\left(\bar{q}_{p} \tau^{I} \gamma^{\mu} q_{r}\right)$ \\
\hline$Q_{\varphi \widetilde{B}}$ & $\varphi^{\dagger} \varphi \widetilde{B}_{\mu \nu} B^{\mu \nu}$ & $Q_{d G}$ & $\left(\bar{q}_{p} \sigma^{\mu \nu} T^{A} d_{r}\right) \varphi G_{\mu \nu}^{A}$ & $Q_{\varphi u}$ & $\left(\varphi^{\dagger} i \stackrel{\leftrightarrow}{D}_{\mu} \varphi\right)\left(\bar{u}_{p} \gamma^{\mu} u_{r}\right)$ \\
\hline$Q_{\varphi W B}$ & $\varphi^{\dagger} \tau^{I} \varphi W_{\mu \nu}^{I} B^{\mu \nu}$ & $Q_{d W}$ & $\left(\bar{q}_{p} \sigma^{\mu \nu} d_{r}\right) \tau^{I} \varphi W_{\mu \nu}^{I}$ & $Q_{\varphi d}$ & $\left(\varphi^{\dagger} i \stackrel{\leftrightarrow}{D}_{\mu} \varphi\right)\left(\bar{d}_{p} \gamma^{\mu} d_{r}\right)$ \\
\hline$Q_{\varphi \widetilde{W} B}$ & $\varphi^{\dagger} \tau^{I} \varphi \widetilde{W}_{\mu \nu}^{I} B^{\mu \nu}$ & $Q_{d B}$ & $\left(\bar{q}_{p} \sigma^{\mu \nu} d_{r}\right) \varphi B_{\mu \nu}$ & $Q_{\varphi u d}$ & $i\left(\widetilde{\varphi}^{\dagger} D_{\mu} \varphi\right)\left(\bar{u}_{p} \gamma^{\mu} d_{r}\right)$ \\
\hline
\end{tabular}

Table C.1: Dimension-six operators other than the four-fermion ones.

\footnotetext{
${ }^{22}$ These tables are taken from [5], by permission of the authors.
} 
All are PTG.

\begin{tabular}{|c|c|c|c|c|c|}
\hline \multicolumn{2}{|r|}{$(\bar{L} L)(\bar{L} L)$} & \multicolumn{2}{|r|}{$(\bar{R} R)(\bar{R} R)$} & \multicolumn{2}{|r|}{$(\bar{L} L)(\bar{R} R)$} \\
\hline$Q_{l l}$ & $\left(\bar{l}_{p} \gamma_{\mu} l_{r}\right)\left(\bar{l}_{s} \gamma^{\mu} l_{t}\right)$ & $Q_{e e}$ & $\left(\bar{e}_{p} \gamma_{\mu} e_{r}\right)\left(\bar{e}_{s} \gamma^{\mu} e_{t}\right)$ & $Q_{l e}$ & $\left(\bar{l}_{p} \gamma_{\mu} l_{r}\right)\left(\bar{e}_{s} \gamma^{\mu} e_{t}\right)$ \\
\hline$Q_{q q}^{(1)}$ & $\left(\bar{q}_{p} \gamma_{\mu} q_{r}\right)\left(\bar{q}_{s} \gamma^{\mu} q_{t}\right)$ & $Q_{u u}$ & $\left(\bar{u}_{p} \gamma_{\mu} u_{r}\right)\left(\bar{u}_{s} \gamma^{\mu} u_{t}\right)$ & $Q_{l u}$ & $\left(\bar{l}_{p} \gamma_{\mu} l_{r}\right)\left(\bar{u}_{s} \gamma^{\mu} u_{t}\right)$ \\
\hline$Q_{q q}^{(3)}$ & $\left(\bar{q}_{p} \gamma_{\mu} \tau^{I} q_{r}\right)\left(\bar{q}_{s} \gamma^{\mu} \tau^{I} q_{t}\right)$ & $Q_{d d}$ & $\left(\bar{d}_{p} \gamma_{\mu} d_{r}\right)\left(\bar{d}_{s} \gamma^{\mu} d_{t}\right)$ & $Q_{l d}$ & $\left(\bar{l}_{p} \gamma_{\mu} l_{r}\right)\left(\bar{d}_{s} \gamma^{\mu} d_{t}\right)$ \\
\hline$Q_{l q}^{(1)}$ & $\left(\bar{l}_{p} \gamma_{\mu} l_{r}\right)\left(\bar{q}_{s} \gamma^{\mu} q_{t}\right)$ & $Q_{e u}$ & $\left(\bar{e}_{p} \gamma_{\mu} e_{r}\right)\left(\bar{u}_{s} \gamma^{\mu} u_{t}\right)$ & $Q_{q e}$ & $\left(\bar{q}_{p} \gamma_{\mu} q_{r}\right)\left(\bar{e}_{s} \gamma^{\mu} e_{t}\right)$ \\
\hline \multirow[t]{4}{*}{$Q_{l q}^{(3)}$} & $\left(\bar{l}_{p} \gamma_{\mu} \tau^{I} l_{r}\right)\left(\bar{q}_{s} \gamma^{\mu} \tau^{I} q_{t}\right)$ & $Q_{e d}$ & $\left(\bar{e}_{p} \gamma_{\mu} e_{r}\right)\left(\bar{d}_{s} \gamma^{\mu} d_{t}\right)$ & $Q_{q u}^{(1)}$ & $\left(\bar{q}_{p} \gamma_{\mu} q_{r}\right)\left(\bar{u}_{s} \gamma^{\mu} u_{t}\right)$ \\
\hline & & $Q_{u d}^{(1)}$ & $\left(\bar{u}_{p} \gamma_{\mu} u_{r}\right)\left(\bar{d}_{s} \gamma^{\mu} d_{t}\right)$ & $Q_{q u}^{(8)}$ & $\left(\bar{q}_{p} \gamma_{\mu} T^{A} q_{r}\right)\left(\bar{u}_{s} \gamma^{\mu} T^{A} u_{t}\right)$ \\
\hline & & $Q_{u d}^{(8)}$ & $\left(\bar{u}_{p} \gamma_{\mu} T^{A} u_{r}\right)\left(\bar{d}_{s} \gamma^{\mu} T^{A} d_{t}\right)$ & $Q_{q d}^{(1)}$ & $\left(\bar{q}_{p} \gamma_{\mu} q_{r}\right)\left(\bar{d}_{s} \gamma^{\mu} d_{t}\right)$ \\
\hline & & & & $Q_{q d}^{(8)}$ & $\left(\bar{q}_{p} \gamma_{\mu} T^{A} q_{r}\right)\left(\bar{d}_{s} \gamma^{\mu} T^{A} d_{t}\right)$ \\
\hline \multicolumn{2}{|c|}{$(\bar{L} R)(\bar{R} L)$ and $(\bar{L} R)(\bar{L} R)$} & & & & \\
\hline$Q_{l e d q}$ & $\left(\bar{l}_{p}^{j} e_{r}\right)\left(\bar{d}_{s} q_{t}^{j}\right)$ & & & & \\
\hline$Q_{q u q d}^{(1)}$ & $\left(\bar{q}_{p}^{j} u_{r}\right) \varepsilon_{j k}\left(\bar{q}_{s}^{k} d_{t}\right)$ & & & & \\
\hline$Q_{q u q d}^{(8)}$ & $\left(\bar{q}_{p}^{j} T^{A} u_{r}\right) \varepsilon_{j k}\left(\bar{q}_{s}^{k} T^{A} d_{t}\right)$ & & & & \\
\hline$Q_{\text {lequ }}^{(1)}$ & $\left(\bar{l}_{p}^{j} e_{r}\right) \varepsilon_{j k}\left(\bar{q}_{s}^{k} u_{t}\right)$ & & & & \\
\hline$Q_{\text {lequ }}^{(3)}$ & $\left(\bar{l}_{p}^{j} \sigma_{\mu \nu} e_{r}\right) \varepsilon_{j k}\left(\bar{q}_{s}^{k} \sigma^{\mu \nu} u_{t}\right)$ & & & & \\
\hline
\end{tabular}

Table C.2: Four-fermion operators conserving baryon number.

\section{Appendix D. SM Equivalence Relations for Dimension-Six Operators}

In the following we use the following notation: $\varphi$ denotes the SM scalar isodoublet; $u, d, e$ denote the right-handed up, down and charged lepton fields, while $l, q$ denote the lepton and quark left-handed doublets. $G_{\mu}^{A},, W_{\mu}^{I}$ and $B_{\mu}$ correspond to the $S U(3)_{c}, S U(2)_{L}$ and $U(1)_{Y}$ gauge fields and $g_{s}, g, g^{\prime}$ the corresponding gauge couplings. $\Gamma_{u, d, e}$ denote the Yukawa-coupling matrices, $\lambda$ the Higgs self-coupling constant; $p, r, s, t$ are family indices and $i, j, k$ denote the $S U(2)_{L}$ indices for $l$ and $q$. $T^{A}=\lambda^{A} / 2$ denote the $S U(3)_{c}$ generators in the fundamental representation, $f^{A B C}$ the corresponding structure constants; and $\varepsilon i j, \varepsilon^{I J K}$ the completely antisymmetric 
tensors in two and three indices. All the operators on the left-hand-side below, except the first, are LG; for ease of reading we group them according to the field content.

Scalars and vectors:

$$
\begin{aligned}
& \left(\varphi^{\dagger} \varphi\right)\left(\varphi^{\dagger} D^{2} \varphi\right) \sim \lambda Q_{\varphi}-\Gamma_{e}^{\dagger} Q_{e \varphi}^{\dagger}-\Gamma_{d}^{\dagger} Q_{d \varphi}^{\dagger}-\Gamma_{u} Q_{u \varphi} \\
& \left(\varphi^{\dagger} i \stackrel{\leftrightarrow}{D}{ }_{\mu}^{I} \varphi\right)^{2} \sim 3 Q_{\varphi \square}-4 \lambda Q_{\varphi}+2\left(\Gamma_{e} Q_{e \varphi}+\Gamma_{d} Q_{d \varphi}+\Gamma_{u} Q_{u \varphi}+\text { h.c. }\right) \\
& \left(D^{2} \varphi\right)^{\dagger} D^{2} \varphi \sim \lambda^{2} Q_{\varphi}-2 \lambda\left(\Gamma_{e} Q_{e \varphi}+\Gamma_{d} Q_{d \varphi}+\Gamma_{u} Q_{u \varphi}+h . c .\right)+\Gamma_{e}^{\dagger} \Gamma_{e} Q_{\ell e}+\Gamma_{d}^{\dagger} \Gamma_{d} Q_{\ell d}+ \\
& +\Gamma_{u}^{\dagger} \Gamma_{u} Q_{\ell u}+\left(\Gamma_{d}^{\dagger} \Gamma_{e} Q_{\ell e d q}+\Gamma_{e} \Gamma_{u} Q_{\text {lequ }}^{(1)}+\Gamma_{d} \Gamma_{u} Q_{\text {quqd }}^{(1)}+\text { h.c. }\right) \text {. } \\
& \left(\varphi^{\dagger} i \stackrel{\leftrightarrow}{D}_{\mu} \varphi\right) \partial_{\nu} B^{\mu \nu} \sim \frac{g^{\prime}}{2}\left(4 Q_{\varphi D}+Q_{\varphi \square}\right)-g^{\prime} \sum_{s}\left(\frac{1}{2} Q_{\varphi \ell}^{(1)}+Q_{\varphi e}-\frac{1}{6} Q_{\varphi q}^{(1)}+\frac{1}{3} Q_{\varphi d}-\frac{2}{3} Q_{\varphi u}\right)_{s} \\
& \left(\varphi^{\dagger} i \stackrel{\leftrightarrow}{D}{ }_{\mu}^{I} \varphi\right) D_{\nu} W^{I \mu \nu} \sim g\left[\frac{3}{2} Q_{\varphi \square}-2 \lambda Q_{\varphi}+\sum_{s}\left(Q_{\varphi \ell}^{(3)}+Q_{\varphi q}^{(3)}\right)_{s}+\right. \\
& \left.+\left(\Gamma_{e} Q_{e \varphi}+\Gamma_{d} Q_{d \varphi}+\Gamma_{u} Q_{u \varphi}+\text { h.c. }\right)\right]
\end{aligned}
$$

Only vectors:

$$
\begin{aligned}
& \left(\partial_{\mu} B^{\mu \nu}\right)^{2} \sim g^{\prime 2}\left[Q_{\varphi D}+\frac{1}{4} Q_{\varphi \square}+\sum_{s}\left(-\frac{1}{2} Q_{\varphi \ell}^{(1)}-Q_{\varphi e}+\frac{1}{6} Q_{\varphi q}^{(1)}-\frac{1}{3} Q_{\varphi d}+\right.\right. \\
& \left.+\frac{2}{3} Q_{\varphi u}\right)_{s}+\sum_{p s}\left(\frac{1}{4} Q_{\ell \ell}^{(1)}+\frac{1}{36} Q_{q q}^{(1)}-\frac{1}{6} Q_{\ell q}^{(1)}+Q_{e e}+\frac{1}{9} Q_{d d}^{(1)}+\frac{4}{9} Q_{u u}^{(1)}+\frac{2}{3} Q_{e d}-\right. \\
& \left.\left.\frac{4}{3} Q_{e u}-\frac{4}{9} Q_{u d}^{(1)}+Q_{\ell e}+\frac{1}{3} Q_{\ell d}-\frac{2}{3} Q_{\ell u}-\frac{1}{3} Q_{q e}-\frac{1}{9} Q_{q d}^{(1)}+\frac{2}{9} Q_{q u}^{(1)}\right)_{p s}\right] \text {. } \\
& \left(D^{\mu} W_{\mu \nu}^{I}\right)^{2} \sim \frac{g^{2}}{4}\left[-4 \lambda Q_{\varphi}+3 Q_{\varphi \square}+2\left(\Gamma_{e} Q_{e \varphi}+\Gamma_{d} Q_{d \varphi}+\Gamma_{u} Q_{u \varphi}+\text { h.c. }\right)+\right. \\
& \left.+2 \sum_{s}\left(Q_{\varphi \ell}^{(3)}+Q_{\varphi \ell}^{(3)}\right)_{s}+\sum_{p s}\left(Q_{\ell \ell}^{(1)}+Q_{q q}^{(3)}+2 Q_{\ell q}^{(3)}\right)_{p s}\right] \text {. } \\
& \left(D^{\mu} G_{\mu \nu}^{A}\right)^{2} \sim g_{s}^{2} \sum_{s t}\left[\frac{1}{4}\left(Q_{q q}^{(3)}+Q_{q q}^{(1)}\right)_{s t t s}+\frac{1}{2}\left(Q_{d d}^{(8)}+Q_{u u}^{(8)}\right)_{s t s t}-\right. \\
& \left.-\frac{1}{6}\left(Q_{q q}^{(1)}+Q_{q d}^{(1)}+Q_{q u}^{(1)}\right)_{s t}+2\left(Q_{q d}^{(8)}+Q_{q u}^{(8)}+Q_{u d}^{(8)}\right)_{s t}\right] \text {. }
\end{aligned}
$$


Fermions and vectors:

$$
\begin{aligned}
& (\overline{i \not D \ell}) i \stackrel{\leftrightarrow}{\not D}(i \not D \ell) \sim \Gamma_{e}^{\dagger} \Gamma_{e}\left[\Gamma_{e} Q_{e \varphi}+\Gamma_{e}^{\dagger} Q_{e \varphi}^{\dagger}+Q_{\varphi e}\right] \\
& (\overline{i \not D e}) i \stackrel{\leftrightarrow}{\not D}(i \not D e) \sim \Gamma_{e} \Gamma_{e}^{\dagger}\left[\Gamma_{e} Q_{e \varphi}+\Gamma_{e}^{\dagger} Q_{e \varphi}^{\dagger}-\frac{1}{2}\left(Q_{\varphi \ell}^{(3)}+Q_{\varphi \ell}^{(1)}\right)\right] \\
& (\overline{i \not D q}) i \overleftrightarrow{\not D}(i \not D q) \sim\left[\Gamma_{u} \Gamma_{u}^{\dagger} \Gamma_{u} Q_{u \varphi}+\Gamma_{d} \Gamma_{d}^{\dagger} \Gamma_{d} Q_{d \varphi}+\text { h.c. }\right]+ \\
& +2\left[\Gamma_{u}^{\dagger} \Gamma_{u} Q_{\varphi u}+\Gamma_{d} \Gamma_{d}^{\dagger} Q_{\varphi d}+2 \Re\left(\Gamma_{u}^{\dagger} \Gamma_{d} Q_{\varphi u d}\right)\right], \\
& (\overline{i \not D d}) i \stackrel{\leftrightarrow D}{(i \not D d}) \sim \Gamma_{d}^{\dagger} \Gamma_{d}\left[\Gamma_{d} Q_{d \varphi}+\Gamma_{d}^{\dagger} Q_{d \varphi}^{\dagger}-\frac{1}{2}\left(Q_{\varphi q}^{(3)}+Q_{\varphi q}^{(1)}\right)\right] \\
& (\overline{i \not D u}) i \overleftrightarrow{\not D}(i \not D u) \sim \Gamma_{u} \Gamma_{u}^{\dagger}\left[\Gamma_{u} Q_{u \varphi}+\Gamma_{u}^{\dagger} Q_{u \varphi}^{\dagger}-\frac{1}{2}\left(Q_{\varphi q}^{(3)}+Q_{\varphi q}^{(1)}\right)\right] \\
& \left(\bar{q} \gamma^{\mu} T^{A} q\right)_{p}\left(D^{\mu} G_{\mu \nu}^{A}\right) \sim g_{s} \sum_{s}\left(Q_{q q}^{(8)}+Q_{q d}^{(8)}+Q_{q u}^{(8)}\right)_{p s}, \\
& \left(\bar{d} \gamma^{\mu} T^{A} d\right)_{p}\left(D^{\mu} G_{\mu \nu}^{A}\right) \sim g_{s} \sum_{s}\left(Q_{q d}^{(8)}+\frac{1}{2} Q_{d d}^{(1)}-\frac{1}{6} Q_{d d}^{(1)}+Q_{u d}^{(8)}\right)_{p s}, \\
& \left(\bar{u} \gamma^{\mu} T^{A} u\right)_{p}\left(D^{\mu} G_{\mu \nu}^{A}\right) \sim g_{s} \sum_{s}\left(Q_{q u}^{(8)}+Q_{u d}^{(8)}+\frac{1}{2} Q_{u u}^{(1)}-\frac{1}{6} Q_{u u}^{(1)}\right)_{p s} . \\
& \left(\bar{q} \gamma^{\mu} \tau^{I} q\right)_{p}\left(D^{\mu} W_{\mu \nu}^{I}\right) \sim \frac{g}{2}\left[\left(Q_{\varphi q}^{(3)}\right)_{p}+\sum_{s}\left(Q_{\ell q}^{(3)}+Q_{q q}^{(3)}\right)_{p s}\right] \\
& \left(\bar{\ell} \gamma^{\mu} \tau^{I} \ell\right)_{p}\left(D^{\mu} W_{\mu \nu}^{I}\right) \sim \frac{\bar{g}}{2}\left[\left(Q_{\varphi \ell}^{(3)}\right)_{p}+\sum_{s}\left(Q_{\ell \ell}^{(3)}+Q_{\ell q}^{(3)}\right)_{p s}\right] \text {. } \\
& \left(\bar{\ell} \gamma^{\mu} \ell\right)_{p} \partial^{\rho} B_{\rho \mu} \sim \frac{g^{\prime}}{2}\left(Q_{\varphi \ell}\right)_{p}+g^{\prime} \sum_{s}\left(-\frac{1}{2} Q_{\ell \ell}^{(1)}+\frac{1}{6} Q_{\ell q}^{(1)}-Q_{\ell e}-\frac{1}{3} Q_{\ell d}+\frac{2}{3} Q_{\ell u}\right)_{p s}, \\
& \left(\bar{q} \gamma^{\mu} q\right)_{p} \partial^{\rho} B_{\rho \mu} \sim \frac{g^{\prime}}{2}\left(Q_{\varphi q}\right)_{p}+g^{\prime} \sum_{s}\left(-\frac{1}{2} Q_{\ell q}^{(1)}+\frac{1}{6} Q_{q q}^{(1)}-Q_{q e}-\frac{1}{3} Q_{q d}^{(1)}+\frac{2}{3} Q_{q u}^{(1)}\right)_{p s}, \\
& \left(\bar{e} \gamma^{\mu} e\right)_{p} \partial^{\rho} B_{\rho \mu} \sim \frac{g^{\prime}}{2}\left(Q_{\varphi e}\right)_{p}+g^{\prime} \sum_{s}\left(-\frac{1}{2} Q_{\ell e}+\frac{1}{6} Q_{q e}-Q_{e e}-\frac{1}{3} Q_{d e}+\frac{2}{3} Q_{u e}\right)_{s p}, \\
& \left(\bar{d} \gamma^{\mu} d\right)_{p} \partial^{\rho} B_{\rho \mu} \sim \frac{g^{\prime}}{2}\left(Q_{\varphi d}\right)_{p}+g^{\prime} \sum_{s}\left(-\frac{1}{2} Q_{\ell d}+\frac{1}{6} Q_{q d}^{(1)}-Q_{e d}-\frac{1}{3} Q_{d d}+\frac{2}{3} Q_{u d}^{(1)}\right)_{s p}, \\
& \left(\bar{u} \gamma^{\mu} u\right)_{p} \partial^{\rho} B_{\rho \mu} \sim \frac{g^{\prime}}{2}\left(Q_{\varphi u}\right)_{p}+g^{\prime} \sum_{s}\left(-\frac{1}{2} Q_{\ell u}+\frac{1}{6} Q_{q u}^{(1)}-Q_{e u}-\frac{1}{3} Q_{u u}+\frac{2}{3} Q_{d u}^{(1)}\right)_{s p}, \\
& \left(\bar{\ell} \sigma^{\mu \nu} i \not D \ell\right) B_{\mu \nu} \sim \Gamma_{e} Q_{e B}, \quad\left(\bar{e} \sigma^{\mu \nu} i \not D e\right) B_{\mu \nu} \sim \Gamma_{e}^{\dagger} Q_{e B}^{\dagger},
\end{aligned}
$$




$$
\begin{aligned}
&\left(\bar{q} \sigma^{\mu \nu} i \not D q\right) B_{\mu \nu} \sim \Gamma_{d} Q_{d B}+\Gamma_{u} Q_{u B}, \\
&\left(\bar{d} \sigma^{\mu \nu} i \not D d\right) B_{\mu \nu} \sim \Gamma_{d}^{\dagger} Q_{d B}^{\dagger}, \\
&\left(\bar{u} \sigma^{\mu \nu} i \not D u\right) B_{\mu \nu} \sim \Gamma_{u}^{\dagger} Q_{u B}^{\dagger} . \\
&\left(\bar{\ell} \sigma^{\mu \nu} \tau^{I} i \not D \ell\right) W_{\mu \nu}^{I} \sim \Gamma_{e} Q_{e W}, \quad\left(\bar{e} \sigma^{\mu \nu} \tau^{I} i \not D e\right) W_{\mu \nu}^{I} \sim \Gamma_{e}^{\dagger} Q_{e W}^{\dagger}, \\
&\left(\bar{q} \sigma^{\mu \nu} \tau^{I} i \not D q\right) W_{\mu \nu}^{I} \sim \Gamma_{d} Q_{d W}+\Gamma_{u} Q_{u W}, \\
&\left(\bar{d} \sigma^{\mu \nu} \tau^{I} i \not D d\right) W_{\mu \nu}^{I} \sim \Gamma_{d}^{\dagger} Q_{d W}^{\dagger}, \\
&\left(\bar{u} \sigma^{\mu \nu} \tau^{I} i \not D u\right) W_{\mu \nu}^{I} \sim \Gamma_{u}^{\dagger} Q_{u W}^{\dagger} . \\
& \\
&\left(\bar{q} \sigma^{\mu \nu} T^{A} i \not D q\right) G_{\mu \nu}^{A} \sim \Gamma_{d} Q_{d G}+\Gamma_{u} Q_{u G}, \\
&\left(\bar{d} \sigma^{\mu \nu} T^{A} i \not D d\right) G_{\mu \nu}^{A} \\
&\left(\bar{u} \sigma^{\mu \nu} T^{A} i \not D u\right) G_{\mu \nu}^{A}
\end{aligned}
$$

Fermions, scalars and vectors:

$$
\begin{aligned}
(\bar{\ell} e) D^{2} \varphi & \sim \lambda Q_{e \varphi}+\frac{1}{2} \Gamma_{e}^{\dagger} Q_{\ell e}-\Gamma_{d}^{\dagger} Q_{\ell e d q}+\Gamma_{u} Q_{\ell e q u}^{(1)}, \\
(\bar{q} d) D^{2} \varphi & \sim \lambda Q_{d \varphi}-\Gamma_{e}^{\dagger} Q_{l e d q}^{\dagger}+\frac{1}{2} \Gamma_{d}^{\dagger} Q_{q d}^{(1)}-\Gamma_{u} Q_{q u q d}^{(1)}, \\
(\bar{q} u) D^{2} \widetilde{\varphi} & \sim \lambda Q_{u \varphi}-\Gamma_{e} Q_{l e q u}^{(1) \dagger}-\Gamma_{d} Q_{q u q d}^{(1)}+\frac{1}{2} \Gamma_{u}^{\dagger} Q_{q u}^{(1)} \\
(\overline{i \not D e}) \varphi^{\dagger}(i \not D \ell) & \sim \Gamma_{e}^{2} Q_{e \varphi}, \\
(\overline{i \not D d}) \varphi^{\dagger}(i \not D q) & \sim \Gamma_{d}^{2} Q_{d \varphi}, \\
(\overline{i \not D u}) \widetilde{\varphi}^{\dagger}(i \not D q) & \sim \Gamma_{u}^{2}(\bar{q} \widetilde{\varphi})\left(\widetilde{\varphi}^{\dagger} \widetilde{\varphi}\right) u=\Gamma_{u}^{2} Q_{u \varphi} .
\end{aligned}
$$

We now define $\left(\mathcal{O}_{\varphi \psi}\right)_{p r} \equiv\left(\varphi^{\dagger} i D_{\mu} \varphi\right)\left(\bar{\psi}_{p} \gamma^{\mu} \psi_{r}\right)$, where $p, r$ are generation indices.

$$
\begin{aligned}
& \left(i D_{\mu} \varphi\right)^{\dagger}\left(\bar{e} \gamma^{\mu} i \not D \ell\right) \sim \Gamma_{e} \mathcal{O}_{\varphi e}^{\dagger}, \quad\left(i D_{\mu} \varphi\right)\left(\bar{\ell} \gamma^{\mu} i \not D e\right) \sim \frac{1}{2} \Gamma_{e}^{\dagger}\left(\mathcal{O}_{\varphi \ell}^{(1)}+\mathcal{O}_{\varphi \ell}^{(3)}\right), \\
& \left(i D_{\mu} \varphi\right)^{\dagger}\left(\bar{d} \gamma^{\mu} i \not D q\right) \sim \Gamma_{d} \mathcal{O}_{\varphi d}^{\dagger}+\Gamma_{u} Q_{\varphi u d}^{\dagger},\left(i D_{\mu} \varphi\right)\left(\bar{q} \gamma^{\mu} i \not D d\right) \sim \frac{1}{2} \Gamma_{d}^{\dagger}\left(\mathcal{O}_{\varphi q}^{(1)}+\mathcal{O}_{\varphi q}^{(3)}\right), \\
& \left(i D_{\mu} \widetilde{\varphi}\right)^{\dagger}\left(\bar{u} \gamma^{\mu} i \not D q\right) \sim \Gamma_{u} \mathcal{O}_{\varphi u}^{\dagger}+\Gamma_{d} Q_{\varphi u d},\left(i D_{\mu} \widetilde{\varphi}\right)\left(\bar{q} \gamma^{\mu} i \not D u\right) \sim \frac{1}{2} \Gamma_{u}^{\dagger}\left(\mathcal{O}_{\varphi q}^{(1)}+\mathcal{O}_{\varphi q}^{(3)}\right) \text {. } \\
& \left(\varphi^{\dagger} \varphi\right)(\bar{\ell} i \not D \ell) \sim \Gamma_{e} Q_{e \varphi}, \quad\left(\varphi^{\dagger} \varphi\right)(\bar{e} i \not D e) \sim \Gamma_{e}^{\dagger} Q_{e \varphi}^{\dagger}, \\
& \left(\varphi^{\dagger} \varphi\right)(\bar{q} i \not D q) \sim \Gamma_{d} Q_{d \varphi}+\Gamma_{u} Q_{u \varphi}, \\
& \left(\varphi^{\dagger} \varphi\right)(\bar{d} i \not D d) \sim \Gamma_{d}^{\dagger} Q_{d \varphi}^{\dagger}, \quad\left(\varphi^{\dagger} \varphi\right)(\bar{u} i \not D u) \sim \Gamma_{u}^{\dagger} Q_{u \varphi}^{\dagger} . \\
& \left(\varphi^{\dagger} \tau^{I} \varphi\right)\left(\bar{\ell} \tau^{I} i \not D \ell\right) \sim \Gamma_{e} Q_{e \varphi}, \quad\left(\varphi^{\dagger} \tau^{I} \varphi\right)\left(\bar{q} \tau^{I} i \not D q\right) \sim \Gamma_{d} Q_{d \varphi}+\frac{1}{2} \Gamma_{u} Q_{u \varphi}
\end{aligned}
$$




\section{References}

[1] See, for example, R. Shankar, "Effective field theory in condensed matter physics," In *Boston 1996, Conceptual foundations of quantum field theory* 47-55 cond-mat/9703210. A. M. Schakel, "Boulevard of broken symmetries: Effective field theories of condensed matter," Hackensack, USA: World Scientific (2008) 390 p. J. Polonyi and A. Schwenk, "Renormalization group and effective field theory approaches to many-body systems," Lect. Notes Phys. 852 (2012).

[2] See, for example, H. Georgi, "Effective field theory," Ann. Rev. Nucl. Part. Sci. 43 (1993) 209. S. Weinberg, "The quantum theory of fields. Vol. 2: Modern applications," Cambridge, UK: Univ. Pr. (1996) 489 p. A. Zee, "Quantum field theory in a nutshell," (2nd ed.), Princeton, NJ: Princeton Univ. Pr. (2010) 576 p. A. G. Grozin, "Heavy quark effective theory," Springer Tracts Mod. Phys. 201 (2004) 1. T. Mannel, "Effective Field Theories in Flavor Physics," Springer Tracts Mod. Phys. 203 (2004) 1. L. Alvarez-Gaume and M. A. Vazquez-Mozo, "An invitation to quantum field theory," Lect. Notes Phys. 839 (2012) 1.

[3] See, for example, A. Pich, "Chiral perturbation theory," Rept. Prog. Phys. 58 (1995) 563 hep-ph/9502366]. S. Scherer and M. R. Schindler, "A Primer for Chiral Perturbation Theory," Lect. Notes Phys. 830 (2012) pp.1.

[4] W. Buchmuller and D. Wyler, Nucl. Phys. B 268 (1986) 621.

[5] B. Grzadkowski, M. Iskrzynski, M. Misiak and J. Rosiek, JHEP 1010 (2010) 085 arXiv:1008.4884 [hep-ph]].

[6] C. Arzt, M. B. Einhorn and J. Wudka, Nucl. Phys. B 433 (1995) 41 hep-ph/9405214.

[7] F. Bonnet, M. B. Gavela, T. Ota and W. Winter, Phys. Rev. D 85, 035016 (2012) arXiv:1105.5140 [hep-ph]]. T. Corbett, O. J. P. Eboli, J. Gonzalez-Fraile and M. C. Gonzalez-Garcia, Phys. Rev. D 87, 015022 (2013) arXiv:1211.4580 [hep-ph]]. G. Belanger, B. Dumont, U. Ellwanger, J. F. Gunion and S. Kraml, JHEP 1302, 053 (2013) [arXiv:1212.5244 [hep-ph]]. C. Grojean, E. E. Jenkins, A. V. Manohar and M. Trott, JHEP 1304 (2013) 016 [arXiv:1301.2588 [hep-ph]]. E. E. Jenkins, A. V. Manohar and M. Trott, arXiv:1305.0017 [hep-ph].

[8] J. Elias-Miro, J. R. Espinosa, E. Masso and A. Pomarol, arXiv:1302.5661 [hepph]. B. Dumont, S. Fichet and G. von Gersdorff, arXiv:1304.3369 [hep-ph]. 
[9] J. Wudka, Int. J. Mod. Phys. A 9 (1994) 2301 hep-ph/9406205].

[10] M. B. Einhorn and J. Wudka, "Effective Field Theory of Higgs Couplings," in preparation.

[11] See, for example, F. Jegerlehner, "The anomalous magnetic moment of the muon," Springer Tracts Mod. Phys. 226, 1 (2008). K. Melnikov and A. Vainshtein, "Theory of the muon anomalous magnetic moment," Springer Tracts Mod. Phys. 216, 1 (2006).

[12] For a recent review, see M. González-Alonso and O. Naviliat-Cuncic, arXiv:1304.1759 [hep-ph].

[13] See, for example, I. I. Bigi and A. I. Sanda, "CP violation," 2nd ed., Cambridge University Press (2009) 506 p. I. B. Khriplovich and S. K. Lamoreaux, "CP violation without strangeness: Electric dipole moments of particles, atoms, and molecules," Berlin, Germany: Springer (1997) 230 p

[14] John T. Moore, Elements of Abstract Algebra, 2nd revised ed. (Collier Macmillan Ltd; London, 1967).

See also http://en.wikipedia.org/wiki/Vector_spaces, and references therein, http://en.wikipedia.org/wiki/Equivalence_relation, and references therein, http://en.wikipedia.org/wiki/Quotient_space_(linear_algebra), and references therein.

[15] H. D. Politzer, Nucl. Phys. B 172 (1980) 349.

[16] C. Arzt, Phys. Lett. B 342 (1995) 189 hep-ph/9304230.

[17] J. Ellison and J. Wudka, Ann. Rev. Nucl. Part. Sci. 48 (1998) 33 hep-ph/9804322.

[18] J. Beringer et al. [Particle Data Group Collaboration], Phys. Rev. D 86 (2012) 010001.

[19] F. del Aguila, A. Aparici, S. Bhattacharya, A. Santamaria and J. Wudka, JHEP 1206 (2012) 146 [arXiv:1204.5986 [hep-ph]].

[20] M. B. Einhorn and J. Wudka, JHEP 0108 (2001) 025 hep-ph/0105035. 